\title{
OONTONG-JAVA PLATEAU
}

\section{V28-238 \\ RC17-176}

These cores were chosen for our initial effort because they typified normal open ocean conditions (see Fig 9; Tables 10, 11).

\section{REFERENCES}

Andrée, M, Beer, J, Oeschger, H, Broecker, W S, Mix, A, Ragano, N, O’Hara, P, Bonani, G, Hofmann, H J, Morenzoni, E, Nessi, M, Suter, M and Wolfli, W, $1984,{ }^{14} \mathrm{C}$ measurements on foraminifera of deep sea core V28-238 and their preliminary interpretation: Nuclear Instruments \& Methods, v B5, p 340-345.

Andrée, M, Beer, J, Oeschger, H, Mix, A, Broecker, W S, Ragano, N, O'Hara, P, Bonani, G, Hofmann, H J, Nessi, M, Suter, M and Wolfli, W, 1985, Accelerator radiocarbon ages on foraminifera separated from deep-sea sediments. Reprinted from, The carbon cycle and atmospheric $\mathrm{CO}_{2}$ : Natural variations Achean to present: Geophys Mono 32, Am Geophys Union.

Peng, T-H and Broecker, W S, 1984, The impacts of bioturbation on the age difference between benthic and planktonic foraminifera in deep sea sediments: Nuclear Instruments \& Methods, v B5, p 346-352.

Shackleton, N J and Opdyke, N D, 1973, Oxygen isotope and paleomagnetic stratigraphy of equatorial Pacific core V28-238, oxygen isotope temperatures and ice volumes on a $10^{5}$ year time scale. Quaternary Research, v 3, p 39-45.
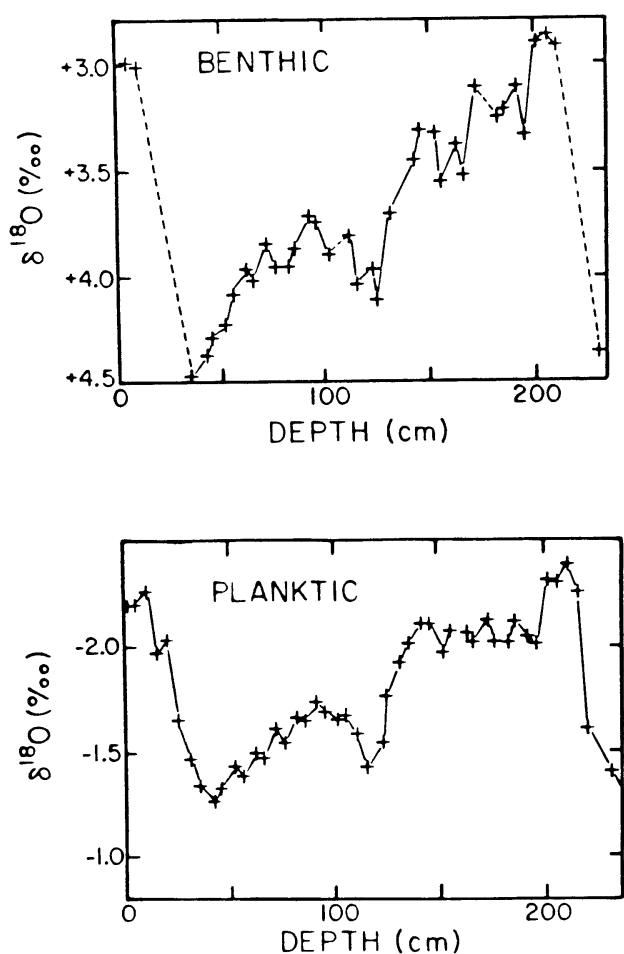

Fig 9. Oxygen isotope records for core V28-238 (Shackleton \& Opdyke, 1973) 
TABLE 10

V28-238 Equatorial Pacific Oontong-Java Plateau Location $\left(01^{\circ} 01^{\prime} \mathrm{N}, 160^{\circ} 29^{\prime} \mathrm{E}\right)$ DEPTH $3120 \mathrm{~m}$

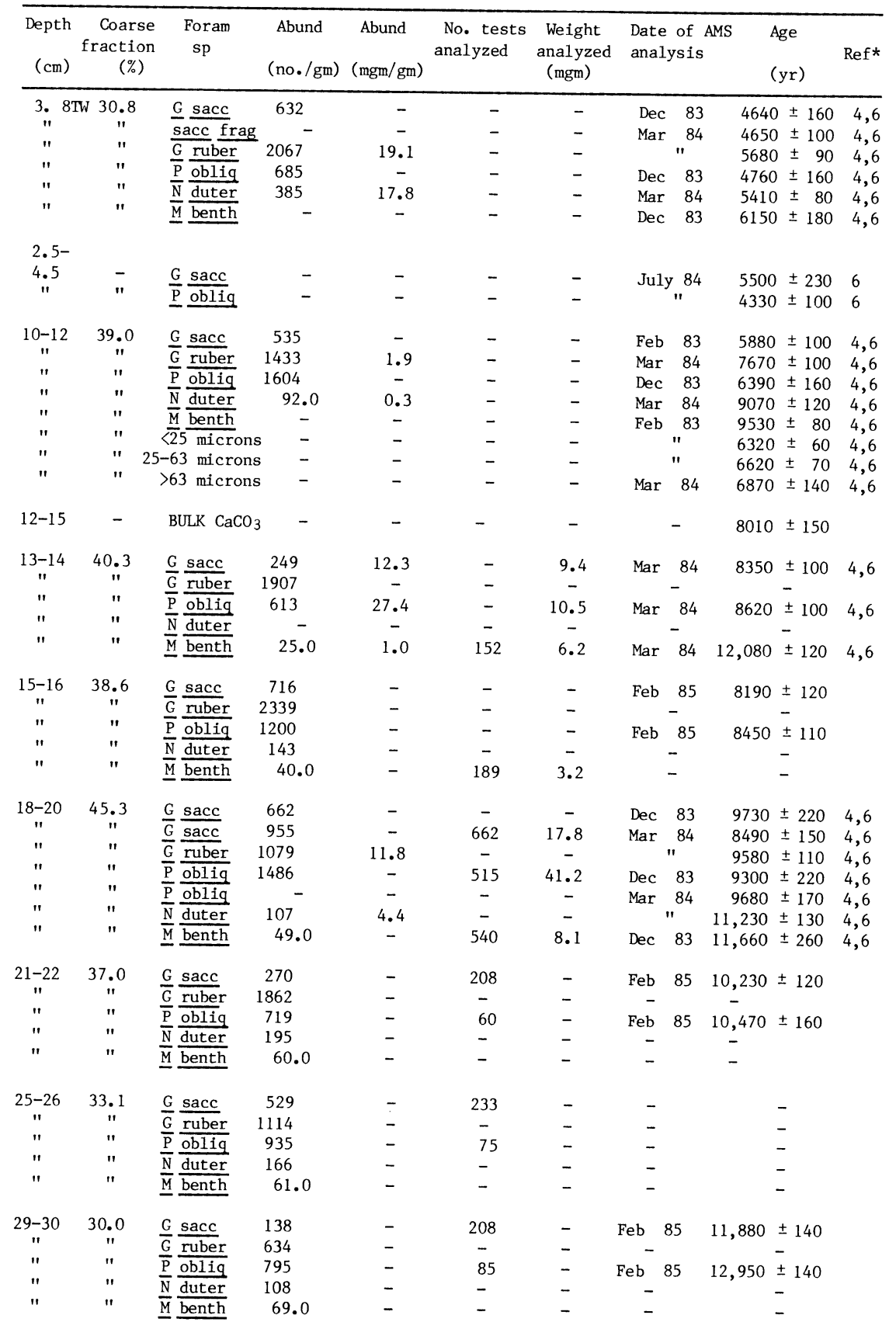


TABLE 10 (cont'd)

\begin{tabular}{|c|c|c|c|c|c|c|c|c|c|c|}
\hline $\begin{array}{l}\text { Depth } \\
(\mathrm{cm})\end{array}$ & $\begin{array}{r}\text { Coarse } \\
\text { fraction } \\
(\%)\end{array}$ & $\begin{array}{l}\text { Foram } \\
\text { sp }\end{array}$ & $\begin{array}{l}\text { Abund } \\
\text { (no./gm) }\end{array}$ & $\begin{array}{l}\text { Abund } \\
\text { (mgm/gm) }\end{array}$ & $\begin{array}{l}\text { No. tests } \\
\text { analyzed }\end{array}$ & $\begin{array}{l}\text { Weight } \\
\text { analyzed } \\
(\mathrm{mgm})\end{array}$ & $\begin{array}{l}\text { Date of AMS } \\
\text { analysis }\end{array}$ & (yr & & Ref* \\
\hline $30-31$ & 25.7 & $\underline{G}$ sacc & 392 & - & 464 & 16.4 & Dec 83 & 11,650 & \pm 260 & 4,6 \\
\hline " & " & $\frac{\bar{p}}{\mathrm{ob} 1 \mathrm{ig}}$ & 1069 & - & 315 & 21.7 & $"$ & 12,680 & \pm 460 & 4,6 \\
\hline " & $"$ & $\bar{M}$ benth & 76.0 & - & 715 & 8.7 & $"$ & 16,140 & \pm 390 & 4,6 \\
\hline $34-35$ & 21.6 & $\underline{G} \underline{\operatorname{sacc}}$ & 96 & 3.40 & 241 & 8.54 & Mar 84 & 13,560 & \pm 220 & 4,6 \\
\hline$"$ & $"$ & $\bar{G} \frac{\text { ruber }}{}$ & 768 & - & - & - & - & & - & \\
\hline " & " & $\underline{\mathrm{p}}$ obliq & 340 & 23.5 & 80 & 8.0 & Feb 85 & 14,340 & \pm 130 & \\
\hline$"$ & " & $\bar{N} \frac{d u}{\text { duter }}$ & 225 & - & - & - & - & & - & \\
\hline " & $"$ & $\bar{M}$ benth & 87.0 & 1.00 & - & - & - & & - & \\
\hline $41-43$ & 24.8 & $\mathrm{G}$ sacc & 93 & - & - & 11.0 & Apr 83 & 17,780 & \pm 390 & 4,6 \\
\hline$"$ & $"$ & P oblig & 648 & - & - & 11.0 & July 83 & 19,620 & \pm 190 & 4,6 \\
\hline$"$ & $"$ & $\bar{N} \frac{\text { duter }}{\text { dut }}$ & 169 & - & - & - & - & & - & \\
\hline$"$ & " & $\bar{M} \frac{d}{\text { benth }}$ & 81.0 & - & 781 & 6.0 & Apr 83 & 20,650 & \pm 220 & 4,6 \\
\hline$"$ & " & $\langle\overline{2} 5$ microns & - & - & - & - & " & 17,800 & \pm 160 & 4,6 \\
\hline$"$ & " 2 & 25-63 microns & - & - & - & - & $"$ & 19,440 & \pm 260 & 4,6 \\
\hline $44-45$ & 21.2 & $\underline{G}$ ruber & 288 & - & - & - & - & & - & \\
\hline$"$ & $"$ & $\mathrm{P} \frac{\mathrm{oblig}}{\mathrm{ob}}$ & 310 & - & - & - & - & & - & \\
\hline$"$ & " & $\bar{N} \frac{\text { duter }}{2}$ & 149 & - & - & - & - & & - & \\
\hline " & " & $\overline{\mathrm{M}}$ benth & 9.00 & - & - & - & - & & - & \\
\hline $45-47$ & 28.8 & $\underline{\mathrm{g} a c c}$ & - & - & - & - & Mar, 84 & 19,620 & \pm 240 & 4,6 \\
\hline$"$ & $"$ & $\overline{\mathrm{G}} \overline{\text { ruber }}$ & 436 & 0.09 & - & - & " & 19,380 & \pm 260 & 4,6 \\
\hline$"$ & $"$ & $\overline{\mathrm{N}}$ duter & 180 & 6.4 & - & - & $"$ & 21,000 & \pm 250 & 4,6 \\
\hline$"$ & $"$ & $\underline{M}$ benth & - & - & - & - & $"$ & 22,110 & \pm 350 & 4,6 \\
\hline $50-51$ & 25.9 & $\underline{\mathrm{G}} \mathrm{sacc}$ & 678 & 21.2 & 458 & 14.5 & Dec, 83 & 19,610 & \pm 620 & 4 \\
\hline$"$ & $"$ & $\overline{\mathrm{G}} \frac{\mathrm{sacc}}{\mathrm{s}}$ & 678 & - & - & - & Mar, 84 & 22,400 & \pm 1180 & 4 \\
\hline$"$ & $"$ & $\overline{\mathrm{G}}$ ruber & 504 & 5.20 & - & - & 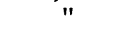 & 21,030 & \pm 280 & 4 \\
\hline$"$ & " & p oblig & 1282 & 75.9 & 433 & 26.9 & Dec, 83 & 22,630 & \pm 1290 & 4 \\
\hline$"$ & $"$ & $\overline{\mathrm{P}} \frac{\mathrm{obliq}}{\mathrm{obl}}$ & - & - & - & - & Mar, 84 & 22,180 & \pm 1090 & 4 \\
\hline$"$ & $"$ & $\bar{N} \frac{1}{\text { duter }}$ & 182 & 0.50 & - & - & & 22,890 & \pm 280 & 4 \\
\hline$"$ & $"$ & $\overline{\mathrm{M}}$ benth & 124 & 1.40 & 678 & 8.1 & Dec, 83 & 22,440 & \pm 690 & 4 \\
\hline 1200 & 21.5 . & $\underline{\mathrm{G}} \mathrm{sacc}$ & 124 & - & - & - & Mar, 84 & & 0140 & 4 \\
\hline$"$ & " & $\overline{\mathrm{p}} \overline{\mathrm{oblig}}$ & - & - & - & - & , & & 1900 & \\
\hline$"$ & $"$ & $\overline{\mathrm{P}}$ obliq & - & - & - & - & Nov, 85 & & 2840 & 4 \\
\hline$"$ & $"$ & $\bar{G} \overline{s a c c}$ & - & - & - & - & & & 6400 & \\
\hline
\end{tabular}

TABLE 11

RC17-176 Equatorial Pacific Oontong-Java Plateau Location $\left(03^{\circ} 45^{\prime} \mathrm{N}, 158^{\circ} 46^{\prime} \mathrm{E}\right)$ Depth $3156 \mathrm{~m}$

\begin{tabular}{|c|c|c|c|c|c|c|c|c|}
\hline $\begin{array}{l}\text { Depth } \\
(\mathrm{cm})\end{array}$ & $\begin{array}{c}\text { Coarse } \\
\text { fraction } \\
(\%)\end{array}$ & $\begin{array}{c}\text { Foram } \\
\mathrm{sp}\end{array}$ & $\begin{array}{l}\text { Abund } \\
\text { (no./gm) }\end{array}$ & $\begin{array}{l}\text { Abund } \\
\text { (mgm/gm) }\end{array}$ & $\begin{array}{l}\text { No. tests } \\
\text { analyzed }\end{array}$ & $\begin{array}{l}\text { Weight } \\
\text { analyzed } \\
(\mathrm{mgm})\end{array}$ & $\begin{array}{l}\text { Date of AMS } \\
\text { analysis }\end{array}$ & $\begin{array}{l}\text { Age } \\
(y r)\end{array}$ \\
\hline $4-6$ & 49.0 & $\underline{\mathrm{G}} \mathrm{sacc}$ & 351 & - & - & - & Dec 83 & $6080 \pm 190$ \\
\hline$"$ & " & $\underline{\bar{P}} \overline{\text { oblig }}$ & 814 & - & - & - & $"$ & $6710 \pm 190$ \\
\hline$"$ & $"$ & $\overline{\mathrm{N}} \overline{\text { duter }}$ & - & - & - & - & $"$ & - \\
\hline$"$ & " & $\overline{\mathrm{M}} \overline{\text { benth }}$ & 25.6 & - & - & - & $"$ & $9400 \pm 220$ \\
\hline
\end{tabular}

